\title{
Artroscopia do joelho de ovinos ${ }^{1}$
}

\author{
Khadije Hette ${ }^{2 \star}$, Sheila C. Rahal ${ }^{3}$, Reinaldo S. Volpi ${ }^{4}$, Danilo Otávio L. \\ Ferreira $^{5}$ e Carlos R. Teixeira ${ }^{6}$
}

\begin{abstract}
Hette K., Rahal S.C.,Volpi R.S., Ferreira D.O.L. \& Teixeira C.R. 2008. [Arthroscopy of the stifle joint in sheep.] Artroscopia do joelho de ovinos. Pesquisa Veterinária Brasileira 28(3):119-123. Departamento de Cirurgia e Anestesiologia Veterinária, Faculdade de Medicina Veterinária e Zootecnia, Universidade Estadual Paulista (Unesp), Campus de Botucatu, Distrito de Rubião Junior s/n, Botucatu, SP 18650-000, Brazil. E-mail: khadijehette@yahoo.com.br

The aim of this study was to evaluate arthroscopically the stifle joint of healthy Santa Ines sheep with different ages, and observe the joint elements according to the established portals. Eighteen Santa Ines sheep were divided into 3 equal groups according to the chronological age: GI - 6 to 8 months old (mean weight $25 \mathrm{~kg}$ ), G II - 2 years old (mean weight $50 \mathrm{~kg}$ ), and G III - 3.5 to 5 years old (mean weight $55 \mathrm{~kg}$ ). Three arthroscopic portals were established: craniolateral primary arthroscopic portal, drainage outflow portal, and craniomedial instrumental portal. The patella, the trochlear groove and ridges, the medial and lateral femoral condyles, and the long digital extensor tendon were easily identified. After removing a great portion of the infrapatellar fat pad using a shaver, the cruciate ligaments and the menisci could be observed, even though with some difficulty. The limbs were flexed and extended to identify the cruciate ligaments. Varus and valgus stress of the joint was used to improve meniscal observation. The difficulty level was inversely proportional to the joint size. In conclusion, the arthroscopic portals allowed identification of the main elements of the knee joint, regardless of animal age, and the arthroscopic method had as advantage early postoperative recovery of the animals.
\end{abstract}

INDEX TERMS: Joint, stifle, arthroscopy, sheep.

RESUMO.- O trabalho teve por objetivo avaliar artroscopicamente a articulação do joelho de ovinos hígidos em diferentes idades, com o intuito de determinar, de acordo

\footnotetext{
${ }^{1}$ Recebido em 17 de outubro de 2007.

Aceito para publicação em 1 de fevereiro de 2008

2 Programa de Pós-Graduação em Medicina Veterinária, área de Cirurgia, Veterinária, Faculdade de Medicina Veterinária e Zootecnia (FMVZ), Universidade Estadual Paulista (Unesp), Cx. Postal 560, Distrito de Rubião Junior s/n, Botucatu, SP 18650-000, Brasil. *Autor para correspondência:khadijehette@yahoo.com.br

${ }^{3}$ Departamento de Cirurgia e Anestesiologia Veterinária, FMVZ, Unesp-Botucatu.

${ }^{4}$ Departamento de Cirurgia e Ortopedia, Faculdade de Medicina, Unesp-Botucatu, Cx. Postal 539, Distrito de Rubião Junior s/n, Botucatu, SP 18650-000, Brasil.

5 Programa de Pós-Graduação em Medicina Veterinária, FMVZ, Unesp-Botucatu.

${ }^{5}$ Departamento de Cirurgia e Anestesiologia Veterinária, FMVZ, Unesp-Botucatu.
}

com os portais estabelecidos, a capacidade de observação das estruturas. Utilizaram-se 18 animais da raça Santa Inês, divididos em três grupos experimentais eqüitativos, conforme a idade cronológica, sendo: Grupo I, 6-8 meses (peso médio de $25 \mathrm{~kg}$ ); Grupo II, 2 anos (peso médio de $50 \mathrm{~kg}$ ); e Grupo III, 3,5-5 anos (peso médio de $55 \mathrm{~kg}$ ). Foram estabelecidos três portais: (1) portal artroscópico primário, craniolateral; (2) portal para fluxo de drenagem; e (3) portal instrumental, craniomedial. Identificaram-se com facilidade a patela, sulco e bordas trocleares, côndilos medial e lateral do fêmur, e tendão do músculo extensor longo dos dedos. Após a remoção da maior parte do coxim gorduroso infrapatelar com emprego do "shaver" foram visibilizados, ainda com dificuldade, os ligamentos cruzados e os meniscos. Para a identificação dos primeiros foram necessárias movimentos de flexão e extensão e para os meniscos manobras de varo e valgo. O grau de dificuldade foi inversamente proporcional ao tamanho da articulação. Sendo assim, foi possível concluir que os 
portais artroscópicos utilizados possibilitaram a identificação das principais estruturas articulares, independente da idade, e o método em si teve por vantagem a rápida recuperação pós-operatória dos animais.

TERMOS DE INDEXAÇÃO: Articulação, joelho, artroscópio, ovino.

\section{INTRODUÇÃO}

O emprego de animais como modelos experimentais para o estudo da patogênese de diversas afecções permitiu um avanço na descoberta de várias modalidades de tratamento, sejam eles clínicos ou cirúrgicos, propiciando o alívio dos sintomas ou erradicação das doenças tanto em humanos como nos animais (Swindle \& Adams 1988).

A articulação femorotibiopatelar é uma das mais sujeitas à carga e das mais lesadas por projetos degenerativos que resultam em impotência funcional (Denny \& Butterworth 2000, Piermattei et al. 2006). Por causa do amplo tamanho, a articulação do joelho de ovinos facilita os estudos relacionados a transplante de menisco, ruptura de ligamento cruzado, transplante de cartilagem e transplante articular (Swindle \& Adams 1988, Jackson et al. 1992, Dürselen et al. 1996, Szomor et al. 2000, Kelly et al. 2006).

O exame artroscópico é realizado tanto para diagnóstico de afecções articulares como para acompanhamento de tratamentos, sejam eles clínicos ou experimentais, evitando muitas vezes nesses últimos a necessidade de eutanásia (Klompmaker \& Veth 1999, Higughi et al. 2000, Chokshi \& Rosen 2004). Além disso, pode ser empregado para reconstrução do ligamento cruzado cranial e caudal, reparo de lesões do menisco, reparo de lesões osteocondrais, indução de artrodese e transplante de menisco, entre outros procedimentos (Allen et al. 1998, Verstraete et al. 1998, Higuchi et al. 2000).

A artroscopia é considerada uma técnica minimamente invasiva, que permite excelente observação e precisão porque as estruturas articulares são magnificadas (Beale et al. 2003). O pequeno tamanho do artroscópio e da bainha permite ao cirurgião colocá-lo em vários compartimentos da articulação (Beale et al. 2003, Piermattei et al. 2006). Se comparada a artrotomia, a artroscopia permite diagnóstico mais preciso de ruptura parcial do ligamento cruzado cranial, alterações da sinóvia e da cartilagem articular, e lesão do menisco (Beale et al. 2003). Os achados podem ser documentados em fotografias, fitas de vídeo ou por meio digital (Taylor 1999, Piermattei et al. 2006).

A principal desvantagem da artroscopia é o custo dos equipamentos e o fato de o procedimento em si despender mais tempo que a artrotomia convencional (Taylor 1999, Rochat 2001). Entretanto, comparativamente à ressonância magnética e à tomografia computadorizada, o valor total é bem inferior (Beale et al. 2003). Adicionalmente, há uma longa curva de aprendizado, necessária para manipulação adequada dos instrumentos (Taylor 1999, Beale et al. 2003). Por exemplo, tem sido sugerido um mínimo de 30 a 50 casos para alcançar habilidade ade- quada para avaliar artroscopicamente o ombro canino, lembrando que esta é uma das articulações mais fáceis de acessar (Rochat 2001).

Visto o número limitado de trabalhos experimentais que utilizaram o exame artroscópico em ovinos (Dürselen et al. 1996, Lu et al. 2000, Szomor et al. 2000, Lu et al. 2006) e a importância desses animais como modelo experimental, o presente trabalho teve por objetivo avaliar a articulação do joelho de ovinos hígidos em diferentes idades, com o intuito de determinar, de acordo com os portais estabelecidos, a capacidade de observação das estruturas.

\section{MATERIAL E MÉTODOS}

A metodologia adotada foi aprovada pela Câmara de Ética em Experimentação Animal da Faculdade de Medicina Veterinária e Zootecnia da Universidade Estadual Paulista (Unesp), Botucatu, SP (protocolo 25/2006).

Foram utilizados 18 ovinos clinicamente hígidos da raça Santa Inês, 12 machos e 6 fêmeas, divididos em três grupos experimentais eqüitativos, de acordo com a idade cronológica, sendo: Grupo I, idade entre 6 e 8 meses (peso médio de $25 \mathrm{~kg}$ ); Grupo II, idade de 2 anos (peso médio de $50 \mathrm{~kg}$ ); e Grupo III, idade entre 3,5 e 5 anos (peso médio de $55 \mathrm{~kg}$ ). Os animais foram vermifugados (Cydectin NF, Fort Dodge, Campinas, SP), numerados de 1 a 18 e alocados em baias de 2,5m x 2,5m (três indivíduos por baia), onde receberam água e feno ad libitum, além do fornecimento diário de ração de manutenção processada com $16 \%$ de proteína. Antes do estudo experimental, estabeleceu-se um período de adaptação de no mínimo 10 dias.

Para realização das artroscopias, se fez uso de anestesia geral inalatória. Após jejum de 24 horas, a pré-medicação com acepromazina (Acepran, Univet, São Paulo, SP) $(0,03 \mathrm{mg} / \mathrm{kg}$ ) foi realizada por via intravenosa. A anestesia foi induzida por via intravenosa com uma combinação de cetamina (Vetaset, Fort Dodge, Campinas, SP) $(5 \mathrm{mg} / \mathrm{kg}$ ) e diazepam (Compaz, Cristália, Itapira, SP) $(0,5 \mathrm{mg} / \mathrm{kg})$ e a manutenção com isofluorano (Isoforine, Cristália, Itapira, SP) vaporizado em 100\% de oxigênio, sob entubação e respiração controlada.

Os membros pélvicos foram tricotomizados e, na seqüência, o animal foi posicionado em decúbito dorsal sobre calha de Claude Bernard, com a mesa inclinada em torno de 30 . Para prevenir o deslizamento, os membros torácicos foram contidos cranialmente à mesa e a região inguinal presa por faixa de esparadrapo. Ambos os membros pélvicos foram mantido livres e na extremidade da calha. A anti-sepsia dos joelhos direito e esquerdo foi realizada com álcool iodado. Atadura de crepe estéril foi utilizada para isolar a região distal do membro, do terço médio da tíbia até os dígitos, sendo posteriormente dispostos os panos de campo operatório. O instrumental artroscópico (camisa, obturador, cânula de fluxo, ótica e "shaver") foi esterilizado com glutaraldeído e a câmera protegida com capa plástica de artroscopia estéril.

Foram estabelecidos três portais: (1) portal artroscópico primário, craniolateral infrapatelar; (2) portal para fluxo de drenagem, craniomedial suprapatelar; e (3) portal instrumental, craniomedial infrapatelar. Com o joelho em extensão efetuouse uma incisão puntiforme com bisturi de lâmina 11 da pele até a cápsula articular, na altura da eminência do platô tibial e lateral ao ligamento patelar. O conjunto obturador e camisa foram 
inseridos através deste orifício e gentilmente direcionado para o portal lateral cranial. Após atravessar a articulação femoropatelar sob a patela, o conjunto foi forçado a sair na pele, que foi incisada, exatamente medial ao tendão do quadríceps. Neste momento, o obturador foi removido permanecendo apenas a camisa. Uma cânula de fluxo $(2,8 \mathrm{~mm}$ de diâmetro e $7 \mathrm{~cm}$ de comprimento) foi acomodada dentro da camisa, que foi então gradualmente tracionada para dentro da articulação. Com delicadeza a cânula de fluxo foi separada da camisa e posicionada dentro do compartimento medial da articulação, adjacente à borda troclear medial do fêmur. A ótica de $2,7 \mathrm{~mm}$ de diâmetro, com comprimento de trabalho de $18 \mathrm{~cm}$ e ângulo de inclinação da objetiva de $30^{\circ}$, foi então inserida dentro da camisa e o fluxo de solução fisiológica de $\mathrm{NaCl}$ 0,9\% iniciado. Após lavagem da articulação, esta foi distendida com a solução para permitir o início da avaliação. O sistema de infusão empregado foi por gravidade, com coluna de líquido entre 2 e $2,5 \mathrm{~m}$ de altura, sem controle de pressão, utilizando frascos com volume de 2 litros.

Na região suprapatelar foram avaliados: a patela, o sulco e as bordas trocleares, o côndilo medial do fêmur, os ligamentos cruzados, o côndilo lateral do fêmur e os meniscos. Para meIhor identificação dos ligamentos cruzados e menisco foi necessário utilizar um "shaver" para remover a gordura do coxim infrapatelar, o qual foi introduzido pelo portal instrumental. A lâmina de "shaver" (Smith \& Nephew Endoscopy, Andover, MA, USA) empregada foi de $2,9 \mathrm{~mm}$ de raio total. Durante o exame, movimentos de flexão, valgo e varo foram efetuados para meIhor visibilizar as estruturas. $\mathrm{O}$ gancho de prova foi algumas vezes introduzido para palpação das estruturas avaliadas. As imagens obtidas foram gravadas em DVD. Após a retirada do instrumental artroscópico, as incisões cirúrgicas foram suturadas com pontos simples isolados utilizando fio mononáilon 2-0.

Ao término do procedimento foi administrado antimicrobiano à base de penicilina e estreptomicina (Multibiótico Reforçado, Vitalfarma Ltda, São Sebastião do Paraíso, MG ) (30.000 Ul/kg de peso vivo em dose única) e meloxicam (Ativus Farmacêutica Ltda, Valinhos, SP) $(0,5 \mathrm{mg} / \mathrm{kg}$ por via intramuscular a cada 24 horas durante três dias). As feridas cirúrgicas foram tratadas com spray prata (Bactrovet Prata, König, Santana de Parnaíba, SP) uma vez ao dia durante quatro dias. Os pontos cutâneos foram removidos após 10 dias de pós-operatório.

\section{RESULTADOS E DISCUSSÃO}

A forma de restrição do animal na mesa operatória foi baseada no preconizado para o exame artroscópico em cães (Beale et al. 2003). A diferença foi que em vez de se colocar uma almofada a vácuo sob o quadril do animal, apenas faixa de esparadrapo prendendo a região inguinal à mesa foi utilizada, a fim de prevenir deslizamento. Além disso, como observado por outros autores (Necas et al. 2002; Beale et al. 2003), a manutenção dos membros pélvicos livres e na extremidade da calha permitiu boa manipulação durante a artroscopia.

Como citado por Chokshi \& Rosen (2004), o posicionamento preciso dos portais artroscópicos é a chave do sucesso do procedimento, caso contrário, a habilidade do cirurgião em manusear os instrumentos pode ser afetada, tornando difícil a observação de algumas partes da articulação do joelho e resultando em lesões iatrogênicas à superfície articular. No presente experimento o artros- cópio foi inserido por acesso craniolateral realizado na altura da eminência do platô tibial e lateral ao ligamento patelar, e portal instrumental medial ao ligamento patelar e na altura do portal craniolateral. Outros experimentos com ovinos também inseriram o artroscópio por acesso lateral e demais instrumentais de apoio pelo acesso medial, sendo que um utilizou o artroscópio para avaliar o efeito da radiofreqüência monopolar em defeitos cartilaginosos da tróclea femoral (Lu et al. 2000) e outro para induzir uma lesão na superfície central do côndilo medial (Lu et al. 2006).

O artroscópio utilizado (ótica de 2,7mm de diâmetro, com comprimento de trabalho de $18 \mathrm{~cm}$ e ângulo de inclinação da objetiva de $30^{\circ}$ ) foi adequado para procedimentos em ovinos, independente do grupo etário. A objetiva de $30^{\circ}$ é a mais empregada por permitir um amplo campo de visão por rotação no seu eixo longitudinal (Rochat 2001, Piermattei et al. 2006). Ainda que um grau de inclinação de lente maior aumente o campo de visão, a orientação do instrumento torna-se mais difícil, a quantidade de luz disponível diminui e a imagem torna-se distorcida (Rochat 2001).

A distensão da cápsula articular foi realizada com solução salina $0,9 \%$, visto estudos não terem observado diferenças entre esse fluido e a solução de Ringer lactato (Rochat 2001). Embora existam sistemas de infusão pressurizada (Rochat 2001, Piermattei et al. 2006), utilizou-se apenas o fluxo por gravidade. Este foi apropriado para manter a distensão articular, utilizando-se uma cânula de fluxo de $2,8 \mathrm{~mm}$ de diâmetro e estando a coluna de líquido a $2 \mathrm{~m}$ de altura.

$O$ ingresso do fluido intra-articular geralmente foi efetuado pelo artroscópio, porém o egresso pode ser obtido por meio de uma agulha ou cânula, ou através do portal instrumental desenvolvido para instrumentação cirúrgica (Piermattei et al. 2006). No presente experimento, foi observado que com o uso do "shaver" a via egressa do fluido pela cânula de fluxo pode ser temporariamente dispensada, visto que o próprio instrumento possui opção de aspiração.

$\mathrm{Na}$ avaliação artroscópica inicial a patela, o sulco e as bordas trocleares, os côndilos medial e lateral do fêmur, e o tendão do músculo extensor longo dos dedos foram identificados com facilidade (Fig.1). A observação dos ligamentos cruzados e meniscos, mesmo com realização de manobras de valgo ou varo, não foi possível devido à infiltração que ocorria, em aproximadamente 10 minutos, dos tecidos moles (sinóvia e adiposo) pela solução salina $0,9 \%$, aliado ao volumoso coxim gorduroso infrapatelar. Após a remoção da maior parte do coxim gorduroso com emprego do "shaver" foram identificados, ainda com dificuldade, os ligamentos cruzados e os meniscos (Fig.1). Para visibilizar os primeiros foram necessários movimentos de flexão e extensão e para os meniscos manobras de varo e valgo. O grau de dificuldade foi inversamente proporcional ao tamanho da articulação, ou seja, maior nos animais de porte menor correspondente ao Grupo I. 


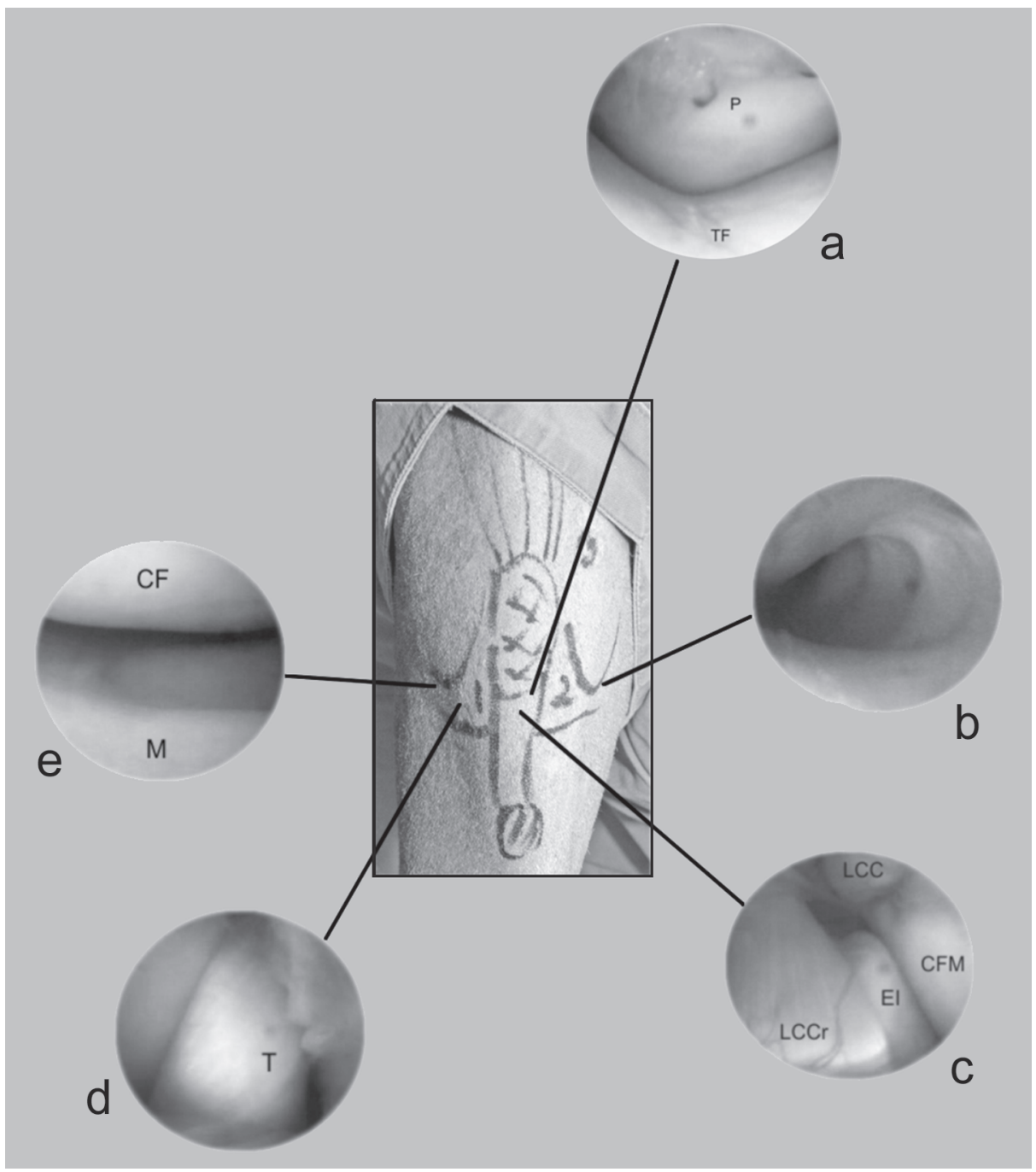

Fig.1. Representação do joelho direito de ovino: o número 1 desenhado na pele do animal corresponde ao portal craniolateral infrapatelar, 2 e 3 representam o portal craniomedial, infra e suprapatelares, respectivamente. a) Visão tangencial da articulação femoropatelar ( $\mathrm{P}=$ patela, TF=tróclea femoral). b) Recesso medial da cápsula articular (b). c) Ligamentos cruzados cranial (LCCr) e caudal (LCC), eminência intercondilar da tíbia (EI) e côndilo femoral medial (CFM). d) tendão do músculo flexor longo dos dedos (T). e) Visão do menisco lateral (M) e côndilo femoral lateral (CF).

Do instrumental básico empregou-se especialmente o gancho de prova que, como referido por Phillips (2003), permitiu sentir a consistência da cartilagem articular, palpar e retrair estruturas. Além disso, o "shaver" motorizado, com lâmina do tipo radial, utilizado para excisar e succionar fragmentos de tecidos moles de forma rápida, precisa e efetiva (Rochat 2001, Phillips 2003, Piermattei et al. 2006), foi essencial para a identificação dos ligamentos cruzados e meniscos. Os ovinos apresentam um grande coxim adiposo infrapatelar que preenche a porção dorsal do espaço articular femorotibial, unindo e obscurecendo o ligamento cruzado cranial (Allen et al. 1998). Sendo assim, da mesma forma que em cães, faz-se necessária a remoção da gordura infrapatelar, a fim de criar um espaço maior para visibilizar as estruturas (Ryssen et al. 2003, Tatarunas \& Matera 2004, Lu et al. 2006).

Como o artroscópio promove magnificação das estruturas, a precisão do exame é maior que a própria artrotomia (Necas et al. 2002, Ralphs \& Whitney 2002). As superfícies cartilagíneas apresentaram aspecto liso e brilhante, porém pela palpação foi observada menor resistência no Grupo I quando comparada aos demais, visto serem os animais jovens.

As complicações encontradas durante a realização do exame artroscópico são variadas. Podem ser citadas incapacidade de adequadamente criar um portal artroscópico ou instrumental, lesão às estruturas intra-articulares, desalojamento prematuro do artroscópio, colapso da cáp- 
sula articular secundário ao excessivo extravasamento de fluido, lesão neurológica, infecção, hemorragia, inabilidade para adequadamente explorar ou tratar a doença articular (Rochat 2001). No presente experimento, um dos problemas mais freqüentes foi o desalojamento prematuro do artroscópio, o que exigia a aplicação do conjunto obturador e camisa para sua reinserção. $O$ fato pode estar associado à curva de aprendizado, necessária para manipulação adequada dos instrumentos (Taylor 1999, Beale et al. 2003), e ao comprimento do mesmo.

Todos os ovinos apresentaram apoio total do membro ao solo após a recuperação anestésica que, em geral, ocorria em torno de meia hora após a extubação. Adicionalmente, não foram observadas complicações pós-operatórias tardias. Isto corrobora com o referido por outros autores que o procedimento artroscópico promove um trauma articular bastante reduzido, resultando em mínimas complicações pós-operatórias ou morbidade (Necas et al. 2002, Beale et al. 2003).

Sendo assim, foi possível concluir que os portais artroscópicos utilizados possibilitaram a identificação das principais estruturas articulares, independente da idade, e o método em si teve por vantagem a rápida recuperação pós-operatória dos animais.

Agradecimentos.- Ao Conselho Nacional de Desenvolvimento Científico e Tecnológico (CNPq) pelo apoio financeiro (Edital Universal, Proc.473637/2004-9) e a FAPESP (Proc.06/59367-7) pela bolsa de mestrado.

\section{REFERÊNCIAS}

Allen M.J., Houton J.E.F., Adams S.B. \& Rushton N. 1998. The surgical anatomy of the stifle joint in sheep. Vet. Surg. 27:596-605.

Beale B.S., Hulse D.A., Schulz K.S. \& Whitney W.O. 2003. Small Animal Arthroscopy. W.B. Saunders, Philadelphia. 231p.

Chokshi B.V. \& Rosen J.E. 2004. Diagnostic arthroscopy of the knee, p.249-259. In: Koval K.J. \& Zuckerman J.D. (ed.), Atlas of Orthopedic Surgery: a multimedia reference. Lippincott Williams and Wilkins, Philadelphia. 554p.

Denny H.R. \& Butterworth S.J. 2000. The stifle, p.512-553. In: (ed.), A guide to Canine and Feline Oorthopaedic Surgery. $4^{\text {th }}$ ed. Blackwell Science, Oxford. 640p.

Dürselen L., Claes L., Ignatius A. \& Rübenacker S. 1996. Comparative animal study of three ligament prostheses for the replacement of the anterior cruciate and medial collateral ligament. Biomaterials 17(10):977-982.

Higuchi H., Kimura M., Shirakura K., Terauchi M. \& Tagagishi K. 2000.
Factors affecting long-term results after arthroscopic partial meniscectomy. Clin. Orthop. Relat. Res. 377:161-168.

Jackson D.W., Mcdevitt C.A., Simon T.M., Arnoczky S.P., Atwell E.A. \& Silvino N.J. 1992. Meniscal transplantation using fresh and cryopreserved allografts. Am. J. Sports Med. 20(6):644-656.

Kelly B.T., Potter H.G., Deng X.H., Pearle A.D., Turner A.S., Warren R.F. \& Rodeo S.A. 2006. Meniscal allograft transplantation in the sheep knee: evaluation of chondroprotective effects. Am. J. Sports Med. 34(90):1464-1478.

Klompmaker J. \& Veth R.P.H. 1999. Animal models of meniscal repair, p.327-347. In: An Y.H. \& Friedman R.J. (ed.), Animal Models in Orthopaedic Research. CRC Press, Boca Raton. 624p.

Lu Y., Hayashi K., Hecht P., Fanton G.S., Thabit III G., Cooley A.J., Edwards R.B. \& Markel M.D. 2000. The effect of monopolar radiofrequency energy on partial-thickness defects of articular cartilage. Arthroscopy 16:527-536.

Lu Y., MarkeL M.D., Swain C. \& Kaplan L.D. 2006. Development of partial thickness articular cartilage injury in an ovine model. J. Orthop. Res. 24:1974-1982.

Necas A., Srnec R. \& Kecova H. 2002. Diagnostic reliability of stifle arthroscopy of pathological changes in cruciate deficient knee. Acta Vet. Brno 71:249-254.

Phillips B.B. 2003. General principles of arthroscopy, p.2497-2514. In: Canale S.T. (ed.), Campbell's Operative Orthopaedics. Vol.3. $10^{\text {th }}$ ed. Mosby, Philadelphia. 1089p.

Piermattei D.L., Flo G.L. \& DeCamp C.E. 2006. The stifle joint, p.562632. In: __ (ed.), Handbook of Small Animal Orthopedics and Fracture Repair. $4^{\text {th }}$ ed. Saunders Elsevier, St Louis. 832p.

Ralphs S.C. \& Whitney W.O. 2002. Arthroscopic evaluation of menisci in dogs with cranial cruciate ligament injuries: 100 cases (1999-2000). J. Am. Vet. Med. Assoc. 221:1601-1604.

Rochat M.C. 2001. Arthroscopy. Vet. Clin. North Am., Small Anim. Clin. 31:761-787.

Ryssen B.V., Bree H.V., Whitney W.O. \& Schulz K.S. 2003. Small animal arthroscopy, p.2285-2312. In: Slatter D.H. (ed.), Textbook of Small Animal Surgery. $3^{\text {rd }}$ ed. W.B. Saunders, Philadelphia. 256p.

Swindle M.M. \& Adams R.J. 1988. Experimental surgery and physiology: induced animal models of human disease. Williams and Wilkins, Baltimore. 350p.

Szomor Z.L., Martin T.E., Bonar F.M.B. \& Murrell G. 2000. The protective effects of meniscal transplantation on cartilage: an experimental study in sheep. J. Bone Joint Surg. Am. 82:80-88.

Tatarunas A.C. \& Matera J.M. 2004. Estudo artroscópico da articulação do joelho em cadáveres de cães. Acta Cir. Bras. 19(4). Disponível em URL: http://www.scielo.br/acb. Acesso em 15.12.2007.

Taylor R.A. 1999. Arthroscopy, p.461-470. In: Tams T.D. (ed.), Small Animal Endoscopy. $2^{\text {nd }}$ ed. Mosby, St Louis. 510p.

Verstraete K.L., Verdonk R., Lootens T., Vertraete P., Rooy J. \& Kunnen M. 1997. Current status and imaging of allograft meniscal transplantation. Eur. J. Rad. 26:16-22. 